\title{
An Octad for Darmstadtium and Excitement for Copernicium
}

\author{
The discovery that copernicium can decay into a new isotope of
} darmstadtium and the observation of a previously unseen excited state of copernicium provide clues to the location of the "island of stability."

\section{By Katherine Wright}

$\Lambda$ holy grail of nuclear physics is to understand the stability of the periodic table's heaviest elements. The problem is, these elements only exist in the lab and are hard to make. In an experiment at the GSI Helmholtz Center for Heavy Ion Research in Germany, researchers have now observed a previously unseen isotope of the heavy element darmstadtium and measured the decay of an excited state of an isotope of another heavy element, copernicium [1]. The results could provide "anchor points" for theories that predict the stability of these heavy elements, says Anton Såmark-Roth, of Lund University in Sweden, who helped conduct the experiments.

A nuclide's stability depends on how many protons $(Z)$ and neutrons $(N)$ it contains. Most elements with $Z$ above 82 (lead) are unstable, although a region of relative stability appears around thorium $(Z=90)$ and uranium $(Z=92)$. Researchers predict that another "island of stability" exists for superheavy elements-somewhere around $Z=114-$ but they have yet to

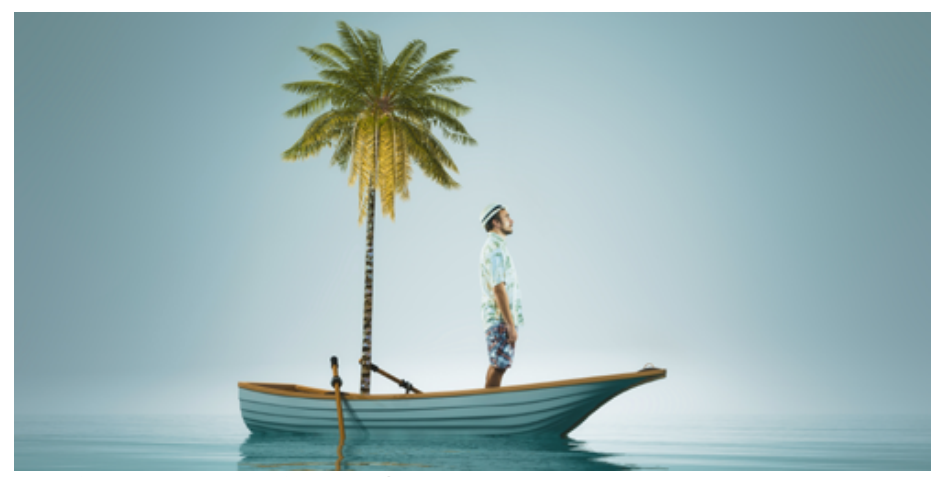

Credit: Orlando Florin Rosu/stock.adobe.com uncover its position.

The team made their discoveries while studying the decay of isotopes of flerovium, which they created by hitting a plutonium target with calcium ions. In their experiments, flerovium-288 $(Z=114, N=174)$ decayed first into copernicium-284 $(Z=112, N=172)$ and then into darmstadtium-280 ( $Z=110$, $N=170$ ), a previously unseen isotope. They also measured an excited state of copernicium-282, another isotope of copernicium. Copernicium-282 is interesting because it contains an even number of protons and neutrons, and researchers had not previously measured an excited state of a superheavy even-even nucleus, Såmark-Roth says.

Katherine Wright is a Senior Editor for Physics.

\section{REFERENCES}

1. A. Såmark-Roth et al., "Spectroscopy along flerovium decay chains: Discovery of ${ }^{280} \mathrm{Ds}$ and an excited state in ${ }^{282} \mathrm{Cn}$," Phys. Rev. Lett. 126, 032503 (2021). 